Instituto Internacional de Investigación y Desarrollo Tecnológico Educativo INDTEC, C.A.

DOI: https://doi.org/10.29394/Scientific.issn.2542-2987.2018.3.10.5.95-114

OAI-PMH: http://www.indteca.com/ojs/index.php/Revista Scientific/oai

Artículo Original / Original Article

\title{
El Docente como Promotor Social y Comunitario en el Marco del Desarrollo Sustentable
}

Autores: Juan Adolfo Salas Ramírez

Universidad Pedagógica Experimental Libertador, UPEL

juansalasgerencia2012@gmail.com

Barinas, Venezuela

Ysmary Del Valle Sayago

Universidad Pedagógica Experimental Libertador, UPEL

ysmarysayago@gmail.com

Barinas, Venezuela

\section{Resumen}

Los métodos de enseñanza y aprendizaje en la actualidad pudiesen estar proyectando un carácter intimidatorio e impositivo, desde luego esto incide en el individuo de tal manera que en mucho de los casos no responde a los intereses particulares, ni a las necesidades que le rodean y por lo tanto compromete, de alguna manera, su proyecto de vida. La Gestión Comunitaria exige espacios académicos donde el recurso humano pueda formular y difundir lo aprendido. En esta perspectiva, la metodología seleccionada fue de una investigación documental apoyada en un estudio monográfico. En consecuencia, se enmarco en el arqueo documental descriptivo, se aplicó igualmente la observación realizada durante los años de servicio educativo, de allí que se propone el diseño de una línea de investigación como apoyo a los programas de postgrado que administra la Universidad Pedagógica Experimental Libertador; denominada El Docente como Promotor Social y Comunitario en el marco del desarrollo sustentable; donde se promueva investigaciones dentro del ámbito de acción e intervención, que generen el análisis y la interpretación de situaciones significativas de la escuelacomunidad-familia, en la búsqueda de alternativas a los problemas ambientales, sociales y/o educativos La orientación de esta apunta hacia las operaciones de investigación de los trabajos de grado producidos por los participantes, permitiendo así la sistematización que orienten los procesos de gestión del docente, con la finalidad de generar soluciones educativas oportunas y pertinentes que impulsen el desarrollo comunitario.

Palabras clave: desarrollo comunitario; desarrollo sostenible; gestión; acción comunitaria.

Fecha de Recepción: 17-07-2018

Fecha de Aceptación: 28-09-2018 
The Teacher as a Social and Community Promoter in the Framework of Sustainable Development

\begin{abstract}
The methods of teaching and learning today could be projecting an intimidating and taxing nature, of course this affects the individual in such a way that in many cases it does not respond to the particular interests, nor to the needs that surround him and for therefore, it compromises, in some way, their life project. Community Management requires academic spaces where human resources can formulate and disseminate what they have learned. In this perspective, the selected methodology was a documentary research supported by a monographic study. Consequently, it is framed in the descriptive documentary archives, the observation made during the years of educational service was applied, from there it is proposed the design of a research line to support the postgraduate programs administered by the Universidad Pedagógica Experimental Libertador; called El Docente as a Social and Community Promoter within the framework of sustainable development; where research is promoted within the scope of action and intervention, which generate the analysis and interpretation of significant situations of the school-community-family, in the search for alternatives to environmental, social and / or educational problems The orientation of this point towards the research operations of the degree works produced by the participants, thus allowing the systematization that guides the processes of teacher management, in order to generate timely and relevant educational solutions that promote community development.
\end{abstract}

Keywords: community development; sustainable development; management; community action.

Date Received: 17-07-2018

Date Acceptance: 28-09-2018 


\section{Introducción}

La investigación es un elemento básico para la solución de los problemas, sobre todo en los contextos humanos ya que la categoría social o sociológica, unida a la educativa cobra más fuerza en la actualidad, visto esto desde la dimensión nacional, regional y local. La corresponsabilidad social de las universidades y otras instituciones educativas pasan por la necesaria visión transdisciplinaria en la que están involucradas, por ende; la concepción científica/epistémica del conocimiento conforma la base primordial en toda investigación.

Surge el interés de proponer el diseño de una línea de investigación como apoyo a los postgrados de la Universidad Pedagógica Experimental Libertador (UPEL), que coadyuve con promover investigaciones dentro del ámbito de acción e intervención, que generen el análisis y la interpretación de situaciones significativas de la escuela-comunidad-familia, en la búsqueda de alternativas a los problemas ambientales, sociales y/o educativos. Desde esta óptica será posible atender las demandas del entorno, de las necesidades sociales y así propiciar formaciones pertinentes desde una concepción ontológica de una universidad-sociedad.

La importancia de la investigación está centrada en la propuesta, la cual podrá contribuir con las nuevas demandas de la educación universitaria, la gestión comunitaria y el desarrollo sustentable. Para Matos (2018): "Dentro de los principales desafíos del desarrollo sustentable está el de propiciar una educación, formación y/o capacitación..." (pág. 235). Cobra igualmente relevancia la construcción de conocimiento desde la óptica de la investigación social y educativa, por cuanto conduce en la mayoría de los casos, a dos argumentos el primero su relación con lo epistemológica de la ciencia social, el segundo con la propia indagación como una de las maneras o prácticas que a partir de una necesidad se produce conocimiento tanto cognitivo como resolutivo; por lo tanto, es la acción el resultado de enterarse de algo y adquirir 
información.

En consecuencia, la investigación que puede ser analizada desde múltiples perspectivas, para Díaz (2006): por ser "un hecho social, cognitivo, discursivo, psicológico, organizacional, debido a que es un proceso de naturaleza compleja en el que interviene factores de muy variada naturaleza" (pág. 93); se puede deducir entonces que la concepción del conocimiento es la base primordial en la investigación y por ende el desarrollo de cualquier sociedad.

Por otra, existen diversos aspectos teóricos a considerar, tales es el caso de la conferencia mundial para la educación superior (UNESCO, 1998): señaló que se debe "promover, generar y difundir conocimientos por medio de la investigación y, como parte de los servicios que ha de prestar a la comunidad, proporcionar las competencias técnicas adecuadas para contribuir al desarrollo cultural, social y económico de las sociedades...," (párr. 20); resulta entonces que es necesario reflexionar sobre la función de las universidades y específicamente la Universidad Pedagógica Experimental Libertador (UPEL) en este sentido, debido a que una de los procesos de la universidad, es concebir la función investigativa como elemento prioritario en los cambios educativos que se desarrollan en el país.

\section{Metodología}

Esta investigación se realizó bajo la modalidad cualitativa, por ello la metodología seleccionada se asienta en una investigación de tipo documental con diseño bibliográfico, enmarcada en un arqueo de carácter analítico e interpretativo. Según Baena (1985): “...es una técnica que consiste en la selección y compilación de información a través de la lectura y crítica de documentos y materiales bibliográficos, bibliotecas, bibliotecas de periódicos, centros de documentación e información" (pág. 23); donde se realizó la indagación de documentos a través de la identificación, selección y organización para acrecentar y ahondar en el contenido abordado que 
pretende presentar algunos insumos teóricos que permitan establecer escenarios de discusión

Por otra parte, Kaufman y Rodríguez (1993), expresan que:

los textos monográficos no necesariamente deben realizarse sobre la base de sólo consultas bibliográficas; se puede recurrir a otras fuentes como, por ejemplo, el testimonio de los protagonistas de los hechos, de testigos calificados, o de especialistas en el tema (pág. 2).

En consecuencia, se infiere de la experiencia de los investigadores como docentes, tutores coordinadores locales y nacionales; en el campo de los subprogramas de postgrado: educación comunitaria, desarrollo de grupos comunitarios, desarrollo comunitario, educación ambiente y desarrollo.

\section{Abordaje de la proposición}

Ubicación disciplinaria de la línea: Campo(s) o Tópico(s): EducaciónComunidad, Ambiente, Promotor Social.

Descripción de la línea: La línea "El docente como promotor social y comunitario en el marco del desarrollo sustentable" constituye una relevante opción para optimizar la producción investigativa y los productos de aplicación comunitaria, específicamente: en los postgrados de la (UPEL), para garantizar en buena medida uno de los fines del hecho investigativo como es la pertinencia social y correspondencia con el contexto que demanda propuestas viables y asimismo, dar respuesta a las necesidades sociales en el seno de las comunidades y su entorno.

Formulación de la Línea: El docente como promotor social y comunitario en el marco del desarrollo sustentable, deriva de la necesidad de: 1). Responder a la Prioridad institucional planteada por el Programa de Acción de la Coordinación Nacional de Investigación; 2). Cumplir con las normas y reglamentos que se exigen en el campo de la investigación y de los Programas 
de Postgrado; 3). Organizar y compartir experiencias de investigadores inmersos en el estudio de los procesos relativos a la gestión comunitaria, ambiente, desarrollo sustentable; 4). Estudiar las relaciones que existen entre el campo educativo, la comunidad, el docente y su entorno; 5). Proponer alternativas de solución a necesidades generales de la comunidad, en ambientes de aprendizaje; 6). Presentar una alternativa novedosa de estudio a profesionales de la Educación con aplicaciones prácticas; 7). Concienciar sobre la necesidad de la organización sistemática de investigaciones útiles en el campo comunitario y su ambiente natural; 8). Producir Proyectos de Investigación, de intervención pedagógica en ambientes de aprendizaje y, de abordaje comunitario para fortalecer el vínculo escuela, familia, comunidad, sociedad y ambiente.

Alcance: En la línea el docente como promotor social y comunitario en el marco del desarrollo sustentable, estudia los procesos que se gestan en las instituciones educativas del sistema educativo venezolano a partir del docente, y que se proyectan a la comunidad en la cual esta se encuentra inmersa y viceversa, donde los actores intervienen directamente con propósitos determinados. El desarrollo de la línea aborda el conocimiento del contexto social-ambiental partiendo de su propia realidad, pretende proponer opciones hacia el mejoramiento real de las comunidades en las dimensiones: pedagógica, cultural, laboral, social, ambiental, entre otras.

La línea aborda áreas temáticas en los diferentes niveles y modalidades del sistema educativo en los cuales se desempeña el docente, interrelacionado con la comunidad de ubicación; los aportes de la línea tienen un alcance práctico, dirigidos a contribuir al mejoramiento de la calidad de vida, vinculando familia-escuela-comunidad. Busca fundamentalmente profundizar con el docente las problemáticas a través de diagnósticos y propuestas de solución, además de la aplicación de los conocimientos comunitarios en la práctica pedagógica, como también precisar el dominio y manejo del conocimiento 
sobre la gestión comunitaria y su problemática.

Misión: En la línea el docente como promotor social y comunitario en el marco del desarrollo sustentable, es una opción para la investigación e intervención comunitaria en ambientes de aprendizajes, cuya misión es suscitar el avance de iniciativas con actores dentro de la escuela o de otras instituciones educativas que directamente lleven al desarrollo de las comunidades en todos sus ámbitos, mediante la solución de sus problemas y necesidades. La consecución de esta misión se logra mediante la propuesta de proyectos específicos y su aplicación de acuerdo con las características propias de cada comunidad a ser abordada, tomando como vínculo; en primer lugar, la escuela como elemento integrador de todo el proceso y, en segundo lugar, a un actor principal de la comunidad: el docente, cuyos conocimientos básicos darán paso en las acciones que fortalezcan la prosperidad comunitaria.

Visión: es una Línea de trabajo institucional como apoyo dentro de los postgrados de la Universidad Pedagógica Experimental Libertador, flexible y abierta a la incorporación de teorías socio-educativas, generadoras de nuevas alternativas y modos de afrontar la realidad en las comunidades, promotora de la formación de una conciencia social hacia la responsabilidad y la participación, creadora de nuevos modelos de abordaje e intervención sociocomunitaria en contextos de aprendizaje, que sirvan de base al mejoramiento y transformación de las colectividades.

Propósito de la línea de investigación: Conscientes de la necesidad de las comunidades de organizarse para lograr su óptimo desarrollo y del papel protagónico del docente en las necesidades sociales de su entorno, esta línea orientada hacia la elección de tópicos sustantivos y adecuada al nivel tiene como propósito el estudio de las estrategias educativas prácticas que van a contribuir a lograr soluciones concretas a problemas de la realidad con el fin de acumular experiencias que sirvan de base a otras investigaciones y así, al 
mejoramiento de la sociedad venezolana en general.

\section{Propósito General:}

4.1. Promover investigaciones dentro del ámbito de acción e intervención, que generen el análisis y la interpretación de situaciones significativas de la escuela-comunidad-familia, en la búsqueda de alternativas a los problemas ambientales, sociales y/o educativos.

\section{Propósitos Específicos:}

5.1. Profundizar estudios de investigación, relacionados con los medios de intervención a las comunidades, partiendo del docente en sus diferentes espacios educativos, locales, regionales y nacionales.

5.2. Relacionar las investigaciones realizadas con el vínculo escuelacomunidad-familia-ambiente.

5.3. Aportar publicaciones que contribuyan al desarrollo de los proyectos de investigación.

5.4. Delinear modelos para el análisis de los procesos comunitarios en Venezuela.

5.5. Colaborar en la comprensión del papel de la escuela en la solución de los problemas de las comunidades donde se desarrollan.

5.6. Orientar las investigaciones hacia focos problemáticos comunes para aprovechar las experiencias previas en los mismos campos y su aplicación a situaciones específicas.

5.7. Discernir en cuanto a las bases teórico-metodológicas de la Línea, su objeto de estudio y sus elementos constitutivos.

\section{Bases teóricas y metodológicas}

\subsection{Fundamentos Generales}

Considerar las bases del quehacer educativo implica la identificación de 
necesidades en diversas esferas- históricas, sociales, metodológicas, entre otros. El estudio de la realidad educativa en Venezuela, permite determinar que la labor diaria de las comunidades genera múltiples problemas característicos de la sociedad actual. De aquí que nace la exigencia de conformar esta Línea de trabajo sobre la base de aspectos como: comportamiento del rol docente en la comunidad, concepciones sobre el desarrollo comunitario, papel de la escuela en el bienestar comunitario, rol de otras instituciones, metodología más adecuada para enfocar la gestión comunitaria.

La urgente necesidad de sistematizar la experiencia obtenida por investigadores en función docente, en relación con la escuela como entidad generadora de la gestión comunitaria, sumada a requisitos institucionales, son las razones académicas que fundamentan la Línea del docente como promotor social y comunitario en el marco del desarrollo sustentable, con un carácter flexible y amplio en armonía a las exigencias de la realidad socioeducativa.

\subsection{Educación Transformadora}

La Línea el docente como promotor social y comunitario en el marco del desarrollo sustentable está inmersa en la educación transformadora, que debe responder a los requerimientos de la producción material en una perspectiva humanista, cooperativa, liberadora, cambiante y, formar en la cultura de la participación ciudadana: la solidaridad social.

Por lo tanto, la línea de investigación presentada busca generar productos que brinden herramientas para superar el marco de la escolarización y la convicción tradicional sobre la enseñanza y así, transformar las condiciones de sus propias comunidades, desdelos ideales de la competitividad, cooperación y la colaboración. Donde el verdadero rol del docente debe trascender las paredes de la escuela, adecuándose a las realidades de su entorno y con una apertura a los nuevos cambios de la 
información y comunicación, concibiendo al individuo el eje fundamental de un proceso transformación global.

La base de la propuesta está fundamentada dentro del Sistema Educativo Bolivariano del año 2008, en el Plan de la Patria 2013-1019; y sobre la concepción de áreas temáticas donde se concrete y materializa la corresponsabilidad de los actores del proceso educativo y así, contribuir a la formación de la cultura participativa desde la perspectiva social, donde escuela comunitaria contribuya a fomentar la calidad de vida de cada uno de sus integrantes.

\subsection{Desarrollo Sustentable}

Para Brundtland (1987): el Desarrollo Sustentable es aquel que "satisface las necesidades de la generación presente sin comprometer la capacidad de las generaciones futuras para satisfacer las propias necesidades" (párr. 1); se puede inferir entonces que es duradero en el tiempo, racional y eficiente en el uso de los recursos y equitativo en los beneficios. Las dimensiones de la Sustentabilidad constituyen el punto único de confluencia de lo social (en su equidad), lo económico (en su prosperidad) y lo ambiental (en integridad ecológica) teniendo como resultado en su interacción de justicia, equidad, ambiente, eficiencia y viabilidad.

\subsection{Bases Metodológicas}

Los fundamentos metodológicos de la Línea el docente como promotor social y comunitario en el marco del desarrollo sustentable se enmarcan en los postulados de naturaleza cualitativa, cuantitativa y cuali-cuantitativa, si bien es cierto que todo aquel que investiga debe ubicar una vía que le permita comprender la realidad para transformarla, la modalidad cualitativa, según Give (2008): “...la investigación cualitativa consiste en un conjunto de prácticas interpretativas que hacen que el mundo sea visible" (pág. 132); se mantiene a 
describir e interpretar determinados contextos y situaciones de la realidad social, de tipo social y cultural; desde el punto de vista ecléctico respecto a enfoques, técnicas, instrumentos, métodos y procedimientos de investigación.

\section{7. Áreas de la línea de investigación}

\subsection{Práctica Pedagógica}

Adecuación del currículo al contexto geográfico, étnico y social, respetando las especificidades comunitarias y favoreciendo la interacción de los actores educativos.

Investigación didáctica para la elaboración de estrategias, proyectos de aprendizajes, recursos para el aprendizaje, para la formación de actitudes favorables contando con la participación de las comunidades y la influencia del entorno sociocultural.

Roles de los diferentes actores del proceso educativo (núcleo familiar, docente, comunidad escolar).

\subsubsection{Interrogantes que genera:}

¿Cuáles son los mecanismos para lograr la adecuación del currículo al contexto social?; ¿Cuál debe ser el rol de los diferentes actores del proceso educativo (docente-alumno-representante-miembros de la comunidad) en el desarrollo curricular?; ¿Cómo optimizar la participación de los padres en el proceso educativo?; ¿Cuáles modalidades permitirían la incorporación de la familia en la elaboración de nuevos recursos y estrategias?; ¿Cómo incorporar a los actores educativos para desarrollar actitudes favorables hacia el aprendizaje?

\subsection{Participación Comunitaria}

Concepción de la escuela como eslabón del poder local, a través de experiencias organizativas con las comunas, consejos comunales, padres y 
representantes, consejo escolar, en la formación de una cultura participativa con conciencia social.

Asesoramiento desde la institución educativa para la sistematización de las actuaciones propias de la comunidad, su entorno y el docente, así como las acciones ligadas a proyectos socioeducativos.

Búsqueda de la transformación con visión innovadora con una acción del estudiante y su entorno.

Acciones por seguir desde las comunidades, priorizando actividades a desarrollar en los problemas más relevantes en los que hay que intervenir; apoyados en un intercambio de experiencias entre los actores comunitarios, teniendo como vínculo a la escuela.

Incorporación de las nuevas tecnologías de comunicación e información corno herramienta facilitadora para la promoción de la participación en la educación virtual y tecnología educativa.

\subsubsection{Interrogantes que genera:}

¿Cómo integrar la escuela a la labor de organizaciones Comunitarias?; ¿Cómo educar para la participación comunitaria?; ¿Cuáles son los mecanismos para convertir el espacio escolar en un eslabón del poder local?; ¿Cómo generar una cultura participativa?; ¿Cuáles son las estrategias para orientar la actividad de las sociedades de padres y representantes hacia el desarrollo comunitario?; ¿Cómo es la participación comunitaria en los programas de intervención y los proyectos sociales?

\subsection{Formación Laboral}

Formación cooperativista, vinculación con experiencias artesanales, desarrollo de tecnologías populares alternativas, apoyo a microempresas para promover el desarrollo local sustentable, escuela y autogestión.

Priorizar el vínculo entre la teoría y la práctica desde una perspectiva 
socioeducativa.

Aportar elementos de formación para la dignificación; de una nueva visión del trabajo.

\subsubsection{Interrogantes que genera:}

¿Cuáles son las estrategias comunitarias para la aplicación de los principios cooperativistas a la solución de problemas?; ¿Cómo difundir las tecnologías alternativas desde la escuela con el apoyo docente?; ¿Cómo promover el desarrollo local sustentable dentro de la comunidad escolar?; ¿Cómo preparar a los jóvenes en ocupaciones no tradicionales en función de divulgar el patrimonio cultural de la comunidad?; ¿Cuáles estrategias desarrolladas desde la escuela mejorarían el nivel de vida de las madres desempleadas?

\subsection{Desarrollo Cultural}

Preservación de los valores propios de la identidad nacional, a través de proyectos culturales, procesos de creación e innovación permanente, creación de agrupaciones artísticas y culturales, rescate de lo originario con una visión de resistencia a los agentes externos.

Incorporación de la cultura de la localidad al currículo como práctica permanente y fortalecimiento de potencialidades de los miembros de la comunidad y con orgullo de nuestras raíces.

\subsubsection{Interrogantes que genera:}

¿Cómo lograr que el docente fomente la participación en actividades culturales?; ¿Cómo fortalecer la identidad cultural dentro de las comunidades?; ¿Cuáles son los procesos que permiten el fortalecimiento de la identidad cultural?; ¿Cuáles estrategias permitirán incorporar la cultura a los Proyectos Pedagógicos? 


\subsection{Problemática Ambiental}

Estudiar problemas del medio ambiente, y los métodos para su protección. Educación ambiental y estrategias para frenar su deterioro. Desarrollo de actitudes conservacionistas. Uso racional de los recursos naturales. Ordenación Territorial. Promover el sistema de valores para ayudar al bienestar y equilibrio entre mente, cuerpo y alma.

En lo que respecta a los modelos económico, político, ambientales; existentes en el momento, y sustentados en los patrones de consumo que determinen una mejor la calidad de vida, exigir a los distintos entes sociales mayor compromiso y responsabilidad en su ejecución.

\subsubsection{Interrogantes que genera:}

¿Cómo sensibilizar a los actores sociales acerca de los problemas ambientales dentro de las comunidades?; ¿Cuáles son las estrategias para el desarrollo de actitudes favorables hacia la protección del ambiente?; ¿Cuáles son las soluciones más pertinentes para disminuir el deterioro ambiental?; ¿Cómo enfrentar el problema de la contaminación dentro de las comunidades, partiendo de la educación escolar?

\subsection{Bienestar Social}

Planificación de estrategias para el progreso de las comunidades a través de la lucha contra la exclusión, combate de la pobreza y mejoramiento de la calidad de vida a través de: proyectos de salud, deporte y recreación, así como programas de intervención grupal dirigidos a la solución de problemas específicos de la comunidad.

Dialogar y reflexionar desde la escuela; sobre la igualdad de oportunidades y condiciones para la integración social, atendiendo a la diversidad multiétnica, intercultural y pluricultural. 


\subsubsection{Interrogantes que genera:}

¿Cuáles estrategias de intervención son más eficaces en la solución de problemas comunitarios?; ¿Cuáles son los criterios de calidad de vida de las distintas comunidades?; ¿Cómo la escuela puede incorporar a todos los agentes comunitarios a favor de la lucha contra el alcoholismo, la drogadicción, la violencia familiar?; ¿Cómo el Docente desde la perspectiva comunitaria propiciaría una mejor calidad de vida?

Figura nro. 1: El presente grafico representa las áreas temáticas de la línea: El Docente como promotor social y comunitario, en el marco del desarrollo sustentable.

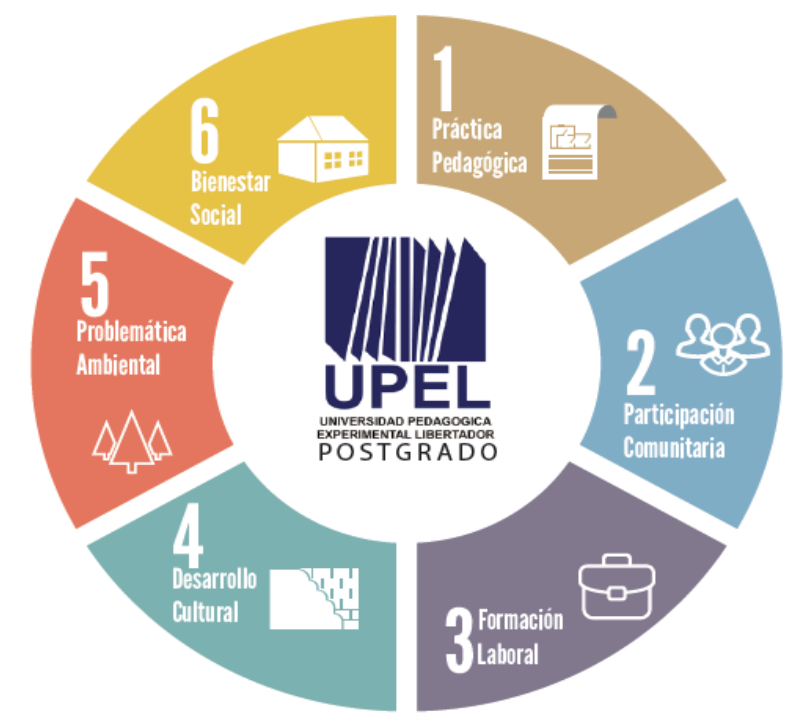

Fuente: Salas (2018).

\section{Conclusiones}

El fundamento de las acciones de la universidad, parte del compromiso que ellas tienen con la escuela-comunidad-familia, por tanto, la calidad en la formación profesional en postgrado debe encaminarse hacia la pertinencia social y así poder ayudar a la sociedad a ser más justa, comprometido, competitivo, saldando entonces la deuda que las instituciones de educación 
superior, tienen con el país. Desde estas perspectivas se exponen las siguientes conclusiones:

La plataforma legal que sustenta la actividad investigativa en el ámbito de la Universidad, es muy completa, al igual que los fundamentos del diseño de la de los Subprogramas de Postgrado. Por tanto, institucionalmente es posible sugerir la implementación de la línea de trabajo: El Docente como Promotor Social y Comunitario en el marco del desarrollo sustentable, que serviría de apoyo en los programas de intervención familia-comunidad-escuela y además a la ejecución los proyectos de trabajo especial de grado, enmarcados en sus áreas socioeducativas y problemas de manifiestos en su entorno.

La investigación es un proceso socializado, sistematizado y orientado a la producción de conocimientos; por lo tanto, permite una participación activa del docente-investigador en el diseño y formulación de líneas de trabajo, partiendo de estrategias participativa y de las nociones de aprender viendo y aprender haciendo.

La línea representa una estrategia que guía la acción del docenteinvestigador, además permitirá satisfacer las funciones docencia-extensiónpostgrado en forma eficiente, eficaz y en concordancia con las realidades socio comunitario.

La línea como elemento estratégico, dinamizador, participo, interventor, ejecutor entre otros, permitirán desarrollar de manera colectiva, lineamientos o acciones hacia la búsqueda de un beneficio común, permitiendo concluir en un producto final.

Las investigaciones en la Universidad tienen un criterio de pertinencia social, por ende, está obligada por así decirlo a satisfacer los intereses de la humanidad, generando en todas sus expresiones una educación humanística con los elevados fundamentos del cocimiento, pero por supuesto con el apoyo incondicional de los actores que lo conforman, que son la expresión viva de la 
realidad: familia-comunidad-docente.

El abordaje del hecho comunitario debe realizarlo un docenteinvestigador con una visión general del significado y el comportamiento de la población, provisto de un lenguaje que le permitan el manejo de términos y conceptos para lograr una lectura social de los fenómenos que ocurren en su contexto. Además, deberá analizar con juicio crítico los diversos enfoques asumidos para diagnosticar, interpretar y aportar soluciones a los problemas sociales del ámbito que le corresponda, asumiendo diferentes roles como un investigador que diagnóstica, un teórico que planifica y un líder que conduce procesos orientados a lograr el beneficio de los grupos comunitarios, pero siempre teniendo como lema del pensamiento ecológico "pensar global, actuar local".

\section{Referencias}

Baena, P. (1985). Metodología de la Investigación. México: Grupo editorial Patria, págs. 5-25.

Brundtland, G. (1987). Nuestro futuro común. (Informe Brundtland). A/42/427. Nairobi, Kenia: Naciones Unidas. Recuperado de:

https://undocs.org/es/A/42/427

Díaz, V. (2006). Formación docente, práctica pedagógica y saber pedagógico. Laurus, 12(Ext), 88-103, ISSN: 1315-883X. Caracas, Venezuela: Universidad Pedagógica Experimental Libertador. Recuperado de: http://www.redalyc.org/articulo.oa?id=76109906

Give, L. (2008). The Sage Encyclopedia of Qualitative Research Methods. USA, United States of America: SEGE Publications, Inc., pp.15-1043.

Kaufman, A., \& Rodríguez, M. (2001). La escuela y los textos. Argentina: Santillana, págs. 11-177.

Matos, Y. (2018). Participación Ciudadana para una Educación Ambiental

Sustentable. Revista Scientific, 3(9), 233-255. Recuperado de: 
https://doi.org/10.29394/Scientific.issn.2542-2987.2018.3.9.12.233-255 UNESCO (1998). Declaración Mundial sobre la Educación Superior en el Siglo XXI: Visión y Acción y Marco de Acción Prioritaria para el Cambio y el Desarrollo de la Educación Superior. París, Francia: UNESCO. Recuperado de:

http://www.unesco.org/education/educprog/wche/declaration spa.htm 


\section{Juan Adolfo Salas Ramírez}

e-mail: juansalasgerencia2012@gmail.com 


\section{Ysmary del Valle Sayago}

e-mail: ysmarysayago@gmail.com

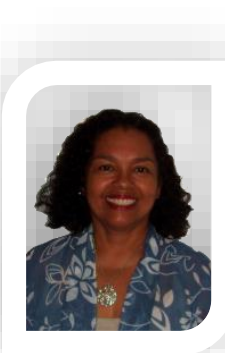

Nacida en Barinas, estado Barinas, Venezuela. Doctora en Ciencias de la Educación (UFT-Lara); Especialista en Desarrollo de grupos Comunitarios (UPEL); Licenciada en Educación Mención Ciencias Naturales Salud y Recreación (UNELLEZ-Barinas); Docente Contratada de la (UPEL-IMPM) núcleo Barinas; Profesora de Pregrado, Extensión y Postgrado; Cátedra de Investigación Cualitativa y Cuantitativa; Seminario de Investigación I-II, Tutoría I-II; Formulación y Evaluación de Proyectos Sociales; Praxis Pedagógica; Tutor y Jurado de trabajos de grado de Especialización y Maestría; Jurado de trabajos de ascenso; Miembro activo del PEII.

El contenido de este manuscrito se difunde bajo una Licencia de Creative Commons ReconocimientoNoComercial-Compartirlgual 4.0 Internacional 